\title{
A LÉVY PROCESS WHOSE JUMPS ARE DRAGGED BY A SPHERICAL DYNAMICAL SYSTEM
}

\author{
BRICE FRANKE, ${ }^{*}$ Ruhr-Universität Bochum
}

\begin{abstract}
We investigate the large scale behaviour of a Lévy process whose jump magnitude follows a stable law with spherically inhomogenous scaling coefficients. Furthermore, the jumps are dragged in the spherical direction by a dynamical system which has an attractor.

Keywords: Scaled Lévy process; dynamical system; large deviation; central limit theorem 2000 Mathematics Subject Classification: Primary 60G51; 60F17
\end{abstract}

\section{Introduction}

In applications one often encounters the situation where the motion of a particle can be decomposed into a deterministic and a random part. The interplay between both dynamics can necessitate a complicated model. Therefore, it is important to be able to reduce its complexity on the base of mathematical reasoning. For example, the path of a sand particle moving on the ground of a turbulent river could be modelled as follows.

Occasionally the particle is lifted into the flow and is dragged to a random location before it sediments back to the ground and waits for its next trip. This transport by saltation was thoroughly investigated and described in Bagnold (1973). According to this work the jumps take place as a result of the tangential force of the moving fluid and the contact with the solid ground. Once the particle has left the ground, its motion follows the turbulent drift imposed by the flow. The Langrangian motion of particles in a turbulent medium has been investigated by Kida (1981), Polvani and Wisdom (1990), and Aref (1990). In Maxey (1990) the chaotic motions of nonspherical particles in laminar flows were described. In recent years quantitative descriptions of the chaotic dynamics were obtained by numerical simulations; see Kawakami and Funakoshi (1999). Our emphasis is to present a model where the abovementioned informations on the chaotic behaviour of the transport can be taken into account.

Since the particle stays in motion only for a relatively short time compared to the period when it is at rest, we will neglect this time and assume that the particle immediately reaches its destination with a jump. This assumption might be questionable since it may be the case that a small particle is lifted into the core of the flow and stays there in suspension for a long time. A more realistic model should have a continuous part. However, we disregard this fact for the sake of simplicity.

After the particle falls back to the ground, it waits for the next jump. For simplicity, we will assume that the mechanism which drives the jumps does not depend on either the location where the particle is waiting for its next jump nor on the history of the motion. It is then reasonable to model the particle as a Lévy process in three-dimensional Euclidean space with a suitable

Received 9 October 2006; revision received 31 July 2007.

* Current address: Institute of Mathematics, Academia Sinica, Taipei, 11529, Taiwan.

Email address: brice.franke@rub.de 
Lévy measure, which is determined by the dynamics of the particle in the flow. The resulting motion of the particle on the ground is then obtained by projection to the plane.

We now want to specify a class of Lévy measures, which is reasonable for this kind of situation. The turbulent behaviour of the vortex which drags the particle during the jump is chaotic; see Aref (1990). Moreover, the motion of an irregular particle due to its shape follows a chaotic dynamic; see Maxey (1990). We will assume that we can decompose the dynamics of the jump into a spherical component and a radial component as follows: while the particle jumps in the radial direction it is dragged simultaneously by the action of the flow in the spherical direction. The resulting jump has the shape of a spiral, when the spherical dynamical system is a rotation. However, more complicated chaotic dynamics on the sphere could be imagined. The underlying motivation for this assumption is that a larger dislocation due to a jump should result in a bigger influence of the dynamical system. Due to the nonlinearity of the problem this is, however, only a rough idealization of the real world phenomenon.

We will assume that the initial state at which the particle enters the spherical dynamical system is randomly and independently selected prior to every jump. In certain states of the flow a departure of the particle is more probable than in others. This can be included in the model by an intensity measure on the sphere which appoints a rate of departures to every part of the state-space. Short trips occur with a higher rate and the distribution of the trips length might have heavy-tails. Therefore, it is natural to model the jump distances by stable increments. However, the index of stability might depend on the state of the spherical dynamical system at which the particle enters the flow.

In this article we define a class of Lévy processes that has the abovementioned characteristics and could serve as a model for a particle which saltates in a fluid flow. Our objective is to prove that this model can be described by simpler ones on large scales when the deterministic spherical dynamical system has an attractor. This model could serve as a starting point for the investigation of multiparticle models for river bed sedimentation. Owing to the importance of sedimentation processes in industry, environmental research, and civil engineering, the transport of particles in fluid flows is a problem that is under constant investigation in physics, engineering, and mathematics. Probabilistic models for sedimentation based on random walks and simple Markov processes can be found, for example, in Pickard and Tory (1977), Gani and Todorovic (1983), Gani (1988), and Ancey et al. (2006).

We now come to the mathematical definition of the Lévy processes studied in this article. Let $v$ be a finite Borel measure on the $(d-1)$-dimensional unit sphere $S^{d-1}$ in $\mathbb{R}^{d}$. Let $\Phi: \mathbb{R}_{0}^{+} \times S^{d-1} \rightarrow S^{d-1}$ be a dynamical system satisfying, for all continuous $f: S^{d-1} \rightarrow \mathbb{R}$ and $\nu$-almost all $\varphi \in S^{d-1}$,

$$
T^{-1} \int_{0}^{T} f\left(\Phi_{t} \varphi\right) \mathrm{d} t \longrightarrow \int_{\mathcal{A}} f \mathrm{~d} \pi \quad \text { as } T \rightarrow \infty,
$$

where $\mathcal{A} \subset S^{d-1}$ is a $\Phi$-invariant subset of $S^{d-1}$ and $\pi$ is an invariant measure on $S^{d-1}$ with support in $\mathcal{A}$. The pair $(\mathcal{A}, \pi)$ is called the Bowen-Ruelle-Sinai attractor for the dynamical system $\left(S^{d-1}, \Phi\right)$; see Buescu (1997, p. 18). Furthermore, let $\alpha: S^{d-1} \rightarrow(0,2)$ be a measurable function having a positive essential infimum $\alpha_{\mathrm{o}}$ with respect to $v$. We use spherical coordinates $(|y|, \hat{y}) \in \mathbb{R}^{+} \times S^{d-1}$ to define the following jump measure:

$$
\mu(A):=\int_{S^{d-1}} \int_{0}^{\infty} \mathbf{1}_{A}\left(|y|, \Phi_{|y|} \hat{y}\right)|y|^{-1-\alpha(\hat{y})} \mathrm{d}|y| v(\mathrm{~d} \hat{y}) .
$$


In the following we want to analyse the large-scale behaviour of the Lévy process $L$ with Lévy exponent

$$
\psi(\xi):=\int_{\mathbb{R}^{d} \backslash\{0\}}\left(\mathrm{e}^{\mathrm{i}\langle\xi, y\rangle}-1-h(|y|) \mathrm{i}\langle\xi, y\rangle \mu(\mathrm{d} y),\right.
$$

where $h: \mathbb{R}^{+} \rightarrow \mathbb{R}$ is a continuous truncation function which is equal to 1 around 0 and such that $y \mapsto\langle\xi, y\rangle h(|y|)$ is bounded for every $\xi \in \mathbb{R}^{d}$. The process $L$ can be defined on a suitable probability space $(\Omega, \mathcal{F}, \mathrm{P})$; see Sato (1999). For $n \in \mathbb{N}$, we introduce the scaled process

$$
L_{t}^{(n)}:=n^{-1 / \alpha_{0}}\left(L_{n t}-c^{(n)} t\right)
$$

where $c^{(n)}$ is a suitable centering having the following expression:

$$
c^{(n)}:=\int_{S^{d-1}} n^{1-(1+\alpha(\hat{z})) / \alpha_{0}} \int_{0}^{\infty}|z|^{-\alpha(\hat{z})} \Phi_{n^{1 / \alpha_{0}}|z|} \hat{z}\left(h(|z|)-h\left(n^{1 / \alpha_{0}}|z|\right)\right) \mathrm{d}|z| v(\mathrm{~d} z) .
$$

Dependent on whether $C_{\mathrm{o}}:=v\left(\alpha=\alpha_{\mathrm{o}}\right)$ is positive or not, we will encounter two different cases. If $C_{\mathrm{o}}>0$, we have the following central limit theorem for the sequence $L^{(n)}$. We denote by $D$ the space of right-continuous paths $\omega:[0, T] \rightarrow \mathbb{R}^{d}$ whose left-limits exist. The Skorohod-topology makes $D$ a separable metric space and it is well known that there exists a modification of $L$ in $D$; see Jacod and Shiryaev (2003).

Theorem 1. If $C_{\mathrm{O}}>0$, the sequence $L^{(n)}$ converges in distribution with respect to the Skorohod-topology toward an $\alpha_{\mathrm{o}}$-stable Lévy process $L^{*}$ determined by the following characteristic exponent:

$$
\psi(\xi)=\int_{\mathbb{R}^{d} \backslash\{0\}}\left(\mathrm{e}^{\mathrm{i}\langle y, \xi\rangle}-1-h(|y|) \mathrm{i}\langle y, \xi\rangle\right) \mu^{*}(\mathrm{~d} y),
$$

with jump measure

$$
\mu^{*}(A)=C_{\mathrm{o}} \int_{0}^{\infty} \int_{\mathcal{A}} \mathbf{1}_{A}(y, \varphi) \pi(\mathrm{d} \varphi)|y|^{-1-\alpha_{0}} \mathrm{~d}|y|
$$

For the case in which $C_{\mathrm{o}}=0$, the scaling is too strong and we obtain convergence in probability toward 0 . It is then important to understand the large deviations for this convergence. Since the increments of the process $L$ have heavy-tails, they fail to satisfy Cramer's condition and the usual approach cannot be used in this setting. A discussion of large deviation theory for processes with heavy-tails can be found in Wentzell (1990, Chapter 6). It turns out that the theory presented there is suitable only for the sequence $L^{(n)}$. The typical behaviour encountered after summation of heavy-tailed random variables is the dominance of a finite number of big jumps on large scales. In the limit, we can recognize only a finite number of events. In order to make these statements precise we need some definitions from Wentzell (1990, Chapter 6). Let

$$
E^{k}:=\left\{\left(x_{1}, \ldots, x_{k}, t_{1}, \ldots, t_{k}\right): 0<t_{1}<\cdots<t_{k}<T, x_{i} \in \mathbb{R}^{d}\right\}
$$

and $D^{k}=\Gamma^{k} E^{k}$, where

$$
\Gamma_{k}: E^{k} \rightarrow D, \quad\left(x_{1}, \ldots, x_{k}, t_{1}, \ldots, t_{k}\right) \mapsto \sum_{i=1}^{k} x_{i} \mathbf{1}_{\left[t_{i}, T\right]}
$$


The space $D^{k}$ contains the paths that possess no more that $k$ jumps and are constant between those jumps. Again we use spherical coordinates to define the measure $\bar{\mu}(\mathrm{d} y)=$ $|y|^{-1-\alpha_{\mathrm{o}}} \mathrm{d}|y| \pi(\mathrm{d} \hat{y})$ on $\mathbb{R}^{d} \backslash\{0\}$. On $D$ we introduce the measure $Q^{k}:=R^{k} \circ \Gamma_{k}^{-1}$, where

$$
R^{k}\left(\mathrm{~d} x_{1}, \ldots, \mathrm{d} x_{k}, \mathrm{~d} t_{1}, \ldots, \mathrm{d} t_{k}\right):=\frac{\bar{\mu}\left(\mathrm{d} x_{1}\right) \cdots \bar{\mu}\left(\mathrm{d} x_{2}\right) \lambda\left(\mathrm{d} t_{1}\right) \cdots \lambda\left(\mathrm{d} t_{k}\right)}{k !} .
$$

The support of $Q^{k}$ is given by $D^{k}$. For a measurable set $A \subset D$, the measure $Q^{k}(A)$ will quantify the proportion of $A$ that the sequence $L^{(n)}$ leaves with rate $g(n)^{k}$. The exact expression of the rate function $g(n)$ is given in Theorem 2, below. The following condition on the regular variation in $\alpha_{\mathrm{o}}$ of the distribution function $F_{\alpha}=v \circ \alpha^{-1}$ is essential in the proof of Theorem 2.

Condition 1. The distribution function $F_{\alpha}$ has a density $F_{\alpha}^{\prime}$ on $\left[\alpha_{0}, 2\right]$ and there exists a $\beta>0$ such that

$$
\frac{F_{\alpha}^{\prime}\left(t x+\alpha_{\mathrm{o}}\right)}{F_{\alpha}^{\prime}\left(t+\alpha_{\mathrm{o}}\right)} \longrightarrow x^{\beta} \quad \text { as } t \rightarrow 0 .
$$

For two subsets $A, B \in D$, we define

$$
d(A, B):=\inf \left\{\left\|\omega_{1}-\omega_{2}\right\|_{\text {sup }}: \omega_{1} \in A, \omega_{2} \in B\right\} .
$$

For a set $A \subset D$, we define $A_{\delta}$ and $A_{-\delta}$ to respectively be the union and intersection of all sets $B$ satisfying $d(A, B)<\delta$.

Theorem 2. If $C_{\mathrm{O}}=0$, assume that Condition 1 holds and let $A$ be a measurable subset of $D$ satisfying

$$
d\left(A, \Gamma_{k-1}\left(E^{k-1}\right)\right)>0 \quad \text { and } \lim _{\delta \downarrow 0} Q^{k}\left(A_{+\delta} \backslash A_{-\delta}\right)=0,
$$

then the following asymptotic holds:

$$
\mathrm{P}\left(L^{(n)} \in A\right)=(g(n))^{k} Q^{k}(A)+o\left((g(n))^{k}\right),
$$

with

$$
g(n):=F_{\alpha}^{\prime}\left(\frac{\alpha_{\mathrm{o}}}{\log n}+\alpha_{\mathrm{o}}\right) \frac{\alpha_{\mathrm{o}}}{\log n} \Gamma(\beta+1) .
$$

In particular, $L^{(n)}$ converges in probability to the zero-function.

In recent years the concept of regular variation has been generalized to $D$-valued random variables and applied to extreme-value theory; see Hult and Lindskog (2005). Let $\gamma>0$ and $\sigma$ be a Borel probability measure on the sphere $S_{D}:=\left\{\omega:\|\omega\|_{\text {sup }}=1\right\}$ in $D$. A $D$-valued random variable $X$ is called regular varying with spectral measure $\sigma$ and tail-index $\gamma$ if, for all $x>0$ and for all measurable $A \subset S_{D}$ with $\sigma(\partial A)=0$, we have

$$
\frac{\mathrm{P}\left(\|X\|_{\text {sup }}>u t, X /\|X\|_{\text {sup }} \in A\right)}{\mathrm{P}\left(\|X\|_{\text {sup }}>u\right)} \longrightarrow t^{-\gamma} \sigma(A) \quad \text { as } u \rightarrow \infty .
$$

The definition of regular variation is quite general and a lot of processes fall into this class. To prove that the process $L$ is regular varying we need a stronger condition on the ergodicity of $\Phi$.

Condition 2. For all $A \subset S^{d-1}$ with $\pi(\partial A)=0$ and $v$-almost all $\varphi \in S^{d-1}$, we have

$$
\frac{1}{T} \int_{0}^{T} \mathbf{1}_{A}\left(\Phi_{s} \varphi\right) \mathrm{d} s \longrightarrow \pi(A) \text { as } t \rightarrow \infty .
$$


Theorem 3. If $C_{\mathrm{o}}>0$ and Condition 2 holds, the Lévy process $L$ is regular varying with tailindex $\alpha_{0}$. Its spectral measure is the distribution of the $S_{D}$-valued process $Z_{t}:=Y \mathbf{1}_{[\tau, 1]}(t)$, where $Y$ is distributed with $\pi_{0}:=\pi / \pi\left(S^{d-1}\right)$ on $S^{d-1}$ and $\tau$ is an independent uniformly distributed random variable on $[0, T]$.

However, it seems that we need strong assumptions on the dynamical system $\Phi$ to prove the regular variation of $L$ when $C_{\mathrm{o}}=0$. The three theorems presented in this note are based on the homogenization of heterogenities on large scales. Similar behaviour was described by the author for stable-like processes with periodic coefficients; see Franke (2006).

\section{The proofs}

The processes $L$ and $L^{(n)}$ are pure-jump processes and, therefore, are determined by the behaviour of their jump measures. The jump measures of the sequence $L^{(n)}$ are given by

$$
\mu^{(n)}(A)=\int_{S^{d-1}} \int_{0}^{\infty} \mathbf{1}_{A}\left(|y|, \Phi_{n^{1 / \alpha_{0}}|y|} \hat{y}\right)|y|^{-1-\alpha(\hat{y})} n^{1-\alpha(\hat{y}) / \alpha_{\mathrm{o}}} \mathrm{d}|y| v(\mathrm{~d} \hat{y}) .
$$

A short computation shows that the Lévy exponent $\psi^{(n)}$ of the centred and scaled process $L_{t}^{(n)}:=n^{-1 / \alpha_{0}}\left(L_{n t}-c^{(n)} t\right)$ is given by

$$
\psi^{(n)}(\xi)=n \psi\left(n^{-1 / \alpha_{0}} \xi\right)=\int_{\mathbb{R}^{d} \backslash\{0\}}\left(\mathrm{e}^{\mathrm{i}\langle\xi, y\rangle}-1-h(|y|) \mathrm{i}\langle\xi, y\rangle\right) \mu^{(n)}(\mathrm{d} y) .
$$

We will need the following lemma.

Lemma 1. Let $f: \mathbb{R}^{+} \times S^{d-1} \rightarrow \mathbb{R}$ be a continuous function such that the function $t \mapsto$ $\sup _{\psi \in S^{d-1}}|f(t, \psi)|$ is Lebesgue integrable on $\mathbb{R}^{+}$. Then, for all sequences $a_{n} \rightarrow \infty$, we have

$$
\int_{0}^{\infty} f\left(t, \Phi_{a_{n} t} \varphi\right) \mathrm{d} t \longrightarrow \int_{0}^{\infty} \int_{\mathcal{A}} f(t, \varphi) \mathrm{d} \pi \mathrm{d} t \quad \text { as } n \rightarrow \infty .
$$

Proof. For a given $\varepsilon>0$, there exists a $T>0$ such that, for all $\varphi \in S^{d-1}$ and $n \in \mathbb{N}$,

$$
\left|\int_{0}^{T} f\left(t, \Phi_{a_{n}} \varphi\right) \mathrm{d} t-\int_{0}^{\infty} f\left(t, \Phi_{a_{n}} \varphi\right) \mathrm{d} t\right|<\varepsilon .
$$

If $T$ is chosen large enough, we also have

$$
\left|\int_{0}^{T} \int_{\mathcal{A}} f(t, \varphi) \pi(\mathrm{d} \varphi) \mathrm{d} t-\int_{0}^{\infty} \int_{\mathcal{A}} f(t, \varphi) \pi(\mathrm{d} \varphi) \mathrm{d} t\right|<\varepsilon .
$$

For large enough $k \in \mathbb{N}$, we have

$$
\left|\int_{0}^{T} \int_{\mathcal{A}} f(t, \varphi) \pi(\mathrm{d} \varphi) \mathrm{d} t-\sum_{j=1}^{k} \frac{T}{k} \int_{\mathcal{A}} f\left(\frac{T j}{k}, \varphi\right) \pi(\mathrm{d} \varphi)\right|<\varepsilon .
$$

Since the function $f$ is uniformly continuous on $[0, T] \times S^{d-1}$, we can choose $k \in \mathbb{N}$ such that

$$
\left|\int_{0}^{T} f\left(t, \Phi_{a_{n} t} \varphi\right) \mathrm{d} t-\sum_{j=1}^{k} \int_{T j / k}^{T(j+1) / k} f\left(\frac{T j}{k}, \Phi_{a_{n} t} \varphi\right) \mathrm{d} t\right|<\varepsilon .
$$


Furthermore, there exists an $n_{0} \in \mathbb{N}$ such that, for all $j \in\{1, \ldots, k\}$ and $n \geq n_{0}$,

$$
\left|\int_{T j / k}^{T(j+1) / k} f\left(\frac{T j}{k}, \Phi_{a_{n} t} \varphi\right) \mathrm{d} t-\frac{T}{k} \int_{\mathcal{A}} f\left(\frac{T j}{k}, \varphi\right) \pi(\mathrm{d} \varphi)\right|<\frac{\varepsilon}{k} .
$$

For this particular choice of $k$ and all $n \geq n_{0}$, we then have

$$
\left|\int_{0}^{T} f\left(t, \Phi_{a_{n} t} \varphi\right) \mathrm{d} t-\int_{0}^{T} \int_{\mathcal{A}} f(t, \varphi) \pi(\mathrm{d} \varphi) \mathrm{d} t\right|<3 \varepsilon .
$$

The choice of $T>0$ now gives

$$
\left|\int_{0}^{\infty} f\left(t, \Phi_{a_{n} t} \varphi\right) \mathrm{d} t-\int_{0}^{\infty} \int_{\mathcal{A}} f(t, \varphi) \pi(\mathrm{d} \varphi) \mathrm{d} t\right|<5 \varepsilon .
$$

This completes the proof.

Proof of Theorem 1. In order to prove the convergence in distribution of $L^{(n)}$ toward $L^{*}$ with respect to the Skorohod-topology we need to prove the following statements (see Jacod and Shiryaev (2003, p. 414)):

1. for all $i, j \in\{1, \ldots, d\}$

$$
\int_{\mathbb{R}^{d} \backslash\{0\}}(h(y))^{2} y_{i} y_{j} \mu^{(n)}(\mathrm{d} y) \longrightarrow \int_{\mathbb{R}^{d} \backslash\{0\}}(h(y))^{2} y_{i} y_{j} \mu^{*}(\mathrm{~d} y) \quad \text { as } n \rightarrow \infty,
$$

2. for all bounded continuous $f: \mathbb{R}^{d} \rightarrow \mathbb{R}$ vanishing in a neighbourhood of 0 ,

$$
\int_{\mathbb{R}^{d} \backslash\{0\}} f(y) \mu^{(n)}(\mathrm{d} y) \longrightarrow \int_{\mathbb{R}^{d} \backslash\{0\}} f(y) \mu^{*}(\mathrm{~d} y) \quad \text { as } n \rightarrow \infty .
$$

Note that the statement on the drift-vector is not mentioned here since, after the centering, the drift-vectors are 0 in our case. Let $g: \mathbb{R}^{d} \rightarrow \mathbb{R}$ be a uniformly continuous, bounded function with the property that, in a neighbourhood of 0 , its absolute value is bounded by the function $x \mapsto|x|^{2}$, then we have

$$
\begin{aligned}
\int_{\mathbb{R}^{d} \backslash\{0\}} g(y) \mu^{(n)}(\mathrm{d} y)= & \int_{S^{d-1}} \int_{0}^{\infty} g\left(|y|, \Phi_{n^{1 / \alpha_{0}}|y|} \hat{y}\right) n^{1-\alpha(\hat{y}) / \alpha_{0}} \mathrm{~d}|y|^{-\alpha(\hat{y})} v(\mathrm{~d} \hat{y}) \\
= & \int_{\left\{\alpha>\alpha_{0}\right\}} \int_{0}^{\infty} g\left(|y|, \Phi_{n^{1 / \alpha_{0}}|y|} \hat{y}\right)|y|^{-1-\alpha(\hat{y})} n^{1-\alpha(\hat{y}) / \alpha_{0}} \mathrm{~d}|y| \nu(\mathrm{d} \hat{y}) \\
& +\int_{\left\{\alpha=\alpha_{0}\right\}} \int_{0}^{\infty} g\left(|y|, \Phi_{n^{1 / \alpha_{0}}|y|} \hat{y}\right)|y|^{-1-\alpha_{0}} \mathrm{~d}|y| v(\mathrm{~d} \hat{y}) .
\end{aligned}
$$

Lebesgue's dominated convergence theorem proves that the first term converges to 0 as $n \rightarrow \infty$. Furthermore, by Lemma 1, we have, for $v$-almost all $\hat{y} \in S^{d-1}$,

$$
\int_{0}^{\infty} g\left(|y|, \Phi_{n^{1 / \alpha_{0}}|y|} \hat{y}\right)|y|^{-1-\alpha_{0}} d|y| \longrightarrow \int_{0}^{\infty} \int_{S^{d-1}} g(|y|, \varphi)|y|^{-1-\alpha_{0}} \pi(\mathrm{d} \varphi) \mathrm{d}|y|
$$

as $n \rightarrow \infty$. Another application of Lebesgue's theorem implies that the second term in the above computation converges toward

$$
\int_{\mathbb{R}^{d} \backslash\{0\}} g(y) \mu^{*}(\mathrm{~d} y)=\int_{\left\{\alpha=\alpha_{0}\right\}} \int_{0}^{\infty} \int_{S^{d-1}} g(|y|, \varphi) \pi(\mathrm{d} \varphi)|y|^{-1-\alpha_{0}} \mathrm{~d}|y| \nu(\mathrm{d} \hat{y}) .
$$


The theorem now follows from the general convergence theorem mentioned at the start of the proof since the above argumentation works for $f: \mathbb{R}^{d} \rightarrow \mathbb{R}$ vanishing at 0 and also for the functions $(h(x))^{2} x_{i} x_{j}$ with $i, j \in\{1, \ldots, d\}$.

Lemma 2. Under the assumptions of Theorem 2 we have

$$
g(n)^{-1} \int_{\alpha_{\mathrm{o}}}^{2} t^{-1-q} n^{1-q / \alpha_{\mathrm{o}}} F_{\alpha}(\mathrm{d} q) \longrightarrow t^{-1-\alpha_{\mathrm{o}}} \quad \text { as } n \rightarrow \infty,
$$

uniformly when $t$ is bounded away from 0 .

Proof. We have

$$
\begin{aligned}
\int_{\alpha_{0}}^{2} t^{-1-q} n^{1-q / \alpha_{0}} F_{\alpha}(\mathrm{d} q) & =\int_{\alpha_{0}}^{2} t^{-1-q} n^{1-q / \alpha_{\mathrm{o}}} F_{\alpha}^{\prime}(q) \mathrm{d} q \\
& =\int_{0}^{2-\alpha_{\mathrm{o}}} t^{-1-r-\alpha_{\mathrm{o}}} n^{-r / \alpha_{\mathrm{o}}} F_{\alpha}^{\prime}\left(r+\alpha_{\mathrm{o}}\right) \mathrm{d} r \\
& =m_{n} \int_{0}^{k_{n}} t^{-1-m_{n} p-\alpha_{\mathrm{o}}} \mathrm{e}^{-p} F_{\alpha}^{\prime}\left(m_{n} p+\alpha_{\mathrm{o}}\right) \mathrm{d} p \\
& =: m_{n} F_{\alpha}^{\prime}\left(m_{n}+\alpha_{\mathrm{o}}\right) t^{-1-\alpha_{\mathrm{o}}} \Gamma(\beta+1)+F(n, t) \\
& =g(n) t^{-1-\alpha_{\mathrm{o}}}+F(n, t),
\end{aligned}
$$

where $k_{n}:=\alpha_{\mathrm{o}}^{-1}\left(2-\alpha_{\mathrm{o}}\right) \log n, m_{n}:=\alpha_{\mathrm{o}} / \log n$, and $F(n, t)$ is the error term. Since we have $k_{n} \rightarrow \infty$ and $m_{n} \rightarrow 0$, as $n \rightarrow \infty$, it follows that

$$
g(n)^{-1} F(n, t)=\Gamma(\beta+1)^{-1} \int_{0}^{k_{n}} t^{-1-m_{n} p-\alpha_{\mathrm{o}}} \mathrm{e}^{-p}\left(\frac{F_{\alpha}^{\prime}\left(m_{n}+\alpha_{\mathrm{o}}\right)}{F_{\alpha}^{\prime}\left(m_{n} p+\alpha_{\mathrm{o}}\right)}-p^{\beta}\right) \mathrm{d} p
$$

converges to 0 uniformly as long as $t$ is bounded away from 0 .

Proof of Theorem 2. According to the conditions presented in Wentzell's book we need to prove the following properties for the sequence $g(n)$ and the measure $\bar{\mu}$; see Wentzell (1990, p. 155).

(i) For all bounded and continuous $f: \mathbb{R}^{d} \rightarrow \mathbb{R}$ vanishing in a neighbourhood of 0 ,

$$
g(n)^{-1} \int_{\mathbb{R}^{d} \backslash\{0\}} f(y) \mu^{(n)}(\mathrm{d} y) \longrightarrow \int_{\mathbb{R}^{d} \backslash\{0\}} f(y) \bar{\mu}(\mathrm{d} y) \quad \text { as } n \rightarrow \infty .
$$

(ii) For all $\delta>0$, there exists a $K(\delta)>0$ such that, for all sufficiently large $n \in \mathbb{N}$, we have

$$
\mu^{(n)}(y ;|y|>\delta) \leq K(\delta) g(n) .
$$

(iii) There exists a constant $K>0$ such that, for all sufficiently large $n \in \mathbb{N}$, we have

$$
\int_{\mathbb{R}^{d} \backslash\{0\}}\left(1 \wedge y^{2}\right) \mu^{(n)}(\mathrm{d} y) \leq K g(n) .
$$


Here we also omitted the condition on the drift-vector, since it is 0 in our case. For a bounded, continuous $f: \mathbb{R}^{d} \rightarrow \mathbb{R}$ vanishing in a neighbourhood of 0 , we have

$$
\begin{aligned}
\int_{S^{d-1}} & \int_{0}^{\infty} f\left(|y|, \Phi_{n^{1 / \alpha_{0}}|y|} \hat{y}\right)|y|^{-1-\alpha(\hat{y})} \mathrm{d}|y| n^{1-\alpha(\hat{y}) / \alpha_{0}} v(\mathrm{~d} \hat{y}) \\
& =\int_{S^{d-1}} \int_{0}^{\infty} \int_{S^{d-1}} f(|y|, \varphi) \pi(\mathrm{d} \varphi)|y|^{-1-\alpha(\hat{y})} \mathrm{d}|y| n^{1-\alpha(\hat{y}) / \alpha_{0}} \nu(\mathrm{d} \hat{y})+E(n, y) \\
& =\int_{\alpha_{0}}^{2} \int_{0}^{\infty} M(|y|)|y|^{-1-q} \mathrm{~d}|y| n^{1-q / \alpha_{0}} F_{\alpha}(\mathrm{d} q)+E(n, y)
\end{aligned}
$$

where

$$
M(|y|):=\int_{S^{d-1}} f(|y|, \varphi) \pi(\mathrm{d} \varphi) .
$$

It follows, from Lemma 1 and the dominated convergence theorem, that the error term

$$
E(n, y)=\int_{\alpha_{0}}^{2} \int_{0}^{\infty}\left(f\left(|y|, \Phi_{n^{1 / \alpha_{0}}|y|} \hat{y}\right)-M(|y|)\right)|y|^{-1-\alpha(\hat{y})} \mathrm{d}|y| n^{1-\alpha(\hat{y}) / \alpha_{0}} v(\mathrm{~d} \hat{y})
$$

converges to 0 as $n \rightarrow \infty$. With Lemma 2 this implies that

$$
g(n)^{-1} \int_{\mathbb{R}^{d} \backslash\{0\}} f(y) \mu^{(n)}(\mathrm{d} y) \longrightarrow \int_{\mathbb{R}^{d} \backslash\{0\}} f(y) \bar{\mu}(\mathrm{d} y) \quad \text { as } n \longrightarrow \infty,
$$

with $\bar{\mu}(\mathrm{d} y)=|y|^{-1-\alpha_{\mathrm{o}}} \mathrm{d}|y| \pi(\mathrm{d} \hat{y})$. This proves statement (i).

Furthermore, for large $n \in \mathbb{N}$, we can use Lemma 2 to show that

$$
\begin{aligned}
\mu^{(n)}(y ;|y|>\delta) & =\int_{\alpha_{0}}^{2} n^{1-q / \alpha_{0}} \int_{\delta}^{\infty}|y|^{-1-q} \mathrm{~d}|y| F_{\alpha}(\mathrm{d} q) \\
& =g(n) \int_{\delta}^{\infty}\left(|y|^{-1-\alpha_{0}} \mathrm{~d}|y|+F(n,|y|) g(n)^{-1}\right) \\
& \leq g(n)(\bar{\mu}(y ;|y|>\delta)+1),
\end{aligned}
$$

where $F(n, y)$ is the error term from the proof of Lemma 2. This proves (ii), with $K(\delta):=$ $\bar{\mu}(y ;|y|>\delta)+1$.

Also, for sufficiently large $n \in \mathbb{N}$, we have

$$
\begin{aligned}
\int_{\mathbb{R}^{d} \backslash\{0\}}\left(1 \wedge y^{2}\right) \mu^{(n)}(\mathrm{d} y) & =g(n) \int_{0}^{\infty}\left(1 \wedge y^{2}\right)\left(|y|^{-1-\alpha_{0}} \mathrm{~d}|y|+g(n)^{-1} F(n,|y|)\right) \\
& \leq g(n)\left(\int_{\mathbb{R}^{d} \backslash\{0\}}\left(1 \wedge y^{2}\right) \bar{\mu}(\mathrm{d} y)+1\right) .
\end{aligned}
$$

Therefore, (iii) follows, with

$$
K:=\int_{\mathbb{R}^{d} \backslash\{0\}}\left(1 \wedge y^{2}\right) \bar{\mu}(\mathrm{d} y)+1 .
$$

Lemma 3. Under Condition 2 we have, for allt $>0, q \in(0,2)$, all $A \subset S^{d-1}$ with $\pi(\partial A)=0$, and for $\nu$-almost all $\varphi \in S^{d-1}$,

$$
\int_{t}^{\infty} \mathbf{1}_{A}\left(\Phi_{u s} \varphi\right) s^{-q} \mathrm{~d} s \longrightarrow \pi(A) \int_{t}^{\infty} s^{-q} \mathrm{~d} s \quad \text { as } u \rightarrow \infty .
$$


Proof. This follows from the following computation:

$$
\begin{aligned}
\lim _{u \rightarrow \infty} \int_{t}^{\infty} \mathbf{1}_{A}\left(\Phi_{u s} \varphi\right) s^{-q} \mathrm{~d} s & =\lim _{u \rightarrow \infty} \int_{0}^{t^{-q}} \int_{t}^{c^{-1 / q}} \mathbf{1}_{A}\left(\Phi_{u s} \varphi\right) \mathrm{d} s \mathrm{~d} c \\
& =\pi(A) \int_{0}^{t^{-q}}\left(c^{-1 / q}-t\right) \mathrm{d} c \\
& =\pi(A) \int_{t}^{\infty} s^{-q} \mathrm{~d} s .
\end{aligned}
$$

Proof of Theorem 3. To prove that the Lévy process $L$ is regular varying with tail-index $\alpha_{\mathrm{o}}$ we need to prove that its jump measure $\mu$ is regular varying in $\mathbb{R}^{d}$ with tail-index $\alpha_{\mathrm{o}}$, i.e.

$$
\frac{\mu(x ;|x| \geq u t, \hat{x} \in A)}{\mu(x ;|x| \geq u)} \longrightarrow t^{-\alpha_{0}} \pi_{\mathrm{o}}(A) \quad \text { as } x \rightarrow \infty
$$

for a suitable probability measure $\pi_{\mathrm{o}}$ on $S^{d-1}$ and all $A \subset S^{d-1}$ with $\sigma_{\mathrm{o}}(\partial A)=0$. The spectral measure $\sigma$ for $L$ is then given by the distribution of the $S_{D}$-valued process $Z_{t}:=Y \mathbf{1}_{[\tau, 1]}$, where $Y$ is distributed with $\pi_{\mathrm{o}}$ on $S^{d-1}$ and $\tau$ is an independent, uniformly distributed random variable on $[0, T]$; see Hult and Lindskog (2005, p. 262). We have

$$
\begin{aligned}
\int_{S^{d-1}} & \int_{u t}^{\infty} \mathbf{1}_{A}\left(\Phi_{|y|} \hat{y}\right)|y|^{-1-\alpha(\hat{y})} \mathrm{d}|y| v(\mathrm{~d} \hat{y}) \\
& =\int_{S^{d-1}} \int_{u t}^{\infty} \pi(A)|y|^{-1-\alpha(\hat{y})} \mathrm{d}|y| v(\mathrm{~d} \hat{y})+E(u) \\
& =\frac{C_{\mathrm{o}}}{\alpha_{\mathrm{o}}}(u t)^{-\alpha_{0}} \pi(A)+\int_{\left\{\alpha>\alpha_{0}\right\}} \int_{u t}^{\infty} \pi(A)|y|^{-1-\alpha(\hat{y})} \mathrm{d}|y| v(\mathrm{~d} \hat{y})+E(u) \\
= & : M(t, u) \pi(A)+F(u)+E(u) .
\end{aligned}
$$

For the first error term, we have

$$
\begin{aligned}
|E(u)| & =\int_{S^{d-1}} o(1, \hat{y}) \int_{u t}^{\infty} \pi(A)|y|^{-1-\alpha(\hat{y})} \mathrm{d}|y| v(\mathrm{~d} \hat{y}) \\
& =u^{-\alpha_{0}} \int_{S^{d-1}} o(1, \hat{y}) \frac{1}{\alpha(\hat{y})} t^{-\alpha(\hat{y})} F_{\alpha}(\mathrm{d} \hat{y}),
\end{aligned}
$$

where $o(1, \hat{y})$ converges to 0 for $v$-almost all $\hat{y}$. Therefore, it follows that $E(u)$ converges toward 0 after division by $M(t, u)$. For an arbitrary $\varepsilon>0$, there exists a $\delta>\alpha_{\mathrm{o}}$ such that $v\left(\alpha_{\mathrm{o}}<\alpha \leq \delta\right) \leq \varepsilon$. It then follows that

$$
\begin{aligned}
|F(u)| \leq & \int_{\left\{\delta \alpha>\alpha_{0}\right\}} \int_{u t}^{\infty} \pi(A)|y|^{-1-\alpha_{0}} \mathrm{~d}|y| v(\mathrm{~d} \hat{y}) \\
& +\int_{\{\alpha>\delta\}} \int_{u t}^{\infty} \pi(A)|y|^{-1-\delta} \mathrm{d}|y| v(\mathrm{~d} \hat{y}) \\
\leq & \varepsilon \frac{1}{\alpha_{\mathrm{o}}}(u t)^{-\alpha_{\mathrm{o}}}+v\left(S^{d-1}\right) \frac{1}{\delta}(u t)^{-\delta} .
\end{aligned}
$$

This implies that the limit of the quotient $F(u) / M(t, u)$ is smaller then $\varepsilon / \alpha_{0}$. Since $\varepsilon>0$ can be arbitrarily small, it follows that $F(u) / M(t, u)$ converges to 0 as $u \rightarrow \infty$. From these 
considerations, it now follows that

$$
\lim _{u \rightarrow \infty} \frac{\mu(x ;|x| \geq u t, \hat{x} \in A)}{\mu(x ;|x| \geq u)}=\lim _{u \rightarrow \infty} \frac{M(t, u) \pi(A)}{M(1, u) \pi\left(S^{d-1}\right)}=t^{-\alpha_{0}} \pi_{\mathrm{o}}(A) .
$$

\section{Acknowledgements}

This research was supported by the DFG, Förderungsnummer: FR2481/1-1. The author also wants to thank Academia Sinica in Taipei for its hospitality.

\section{References}

Ancey, C., Böhm, T., Jodeau, M. And Frey P. (2006). Statistical description of sediment transport experiments. Physical Reviews E 74, 011302.

Aref, H. (1990). Chaotic advection of fluid particles. Philos. Trans. Physical Sci. Eng. 333, 273-288.

BAGnOld, R. (1973). The nature of saltation and bed-load transport in water. Proc. R. Soc. London A 332, $473-504$.

Buescu, J. (1997). Exotic Attractors. From Liapunov Stablity to Riddled Basins (Progr. Math. 153). Birkhäuser, Basel.

Franke, B. (2006). The scaling limit behaviour of periodic stable-like processes. Bernoulli 12, 551-570.

GANI, J. (1988). A correlated random walk for the transport and sedimentation of particles. In A Celebration of Applied Probability (J. Appl. Prob. Spec. Vol. 25A) Applied Probability Trust, Sheffield, pp. 335-346.

Gani, J. And Todorovic, P. (1983). A model for the transport of solid particles in a fluid flow. Stoch. Process. Appl. 14, 1-17.

Jacod, J. And Shiryaev, A. (2003). Limit Theorems for Stochastic Processes, 2nd edn. Springer, Berlin.

Hult, H. ANd Lindskog, F. (2005). Extremal behaviour of regularly varying stochastic processes. Stoch. Process. Appl. 115, 249-274.

KaWAKami, A. And Funakoshi, M. (1999). Chaotic motion of fluid particles around a rotating elliptic vortex in a linear shear flow. Fluid Dynamics Res. 25, 167-193.

KIDA, S. (1981). Motion of an elliptic vortex in a uniform shear flow. J. Physical Soc. Japan 50, 3517-3520.

Maxey, M. (1990). On the advection of spherical and non-spherical particles in a non-uniform flow. Philos. Trans. Physical Sci. Eng. 333, 289-307.

Pickard, D. And Tory, E. (1977). A Markov model for sedimentation. J. Math. Anal. Appl. 60, 349-369.

Polvani, L. AND Wisdom, J. (1990). Chaotic Lagrangian trajectories around an elliptic vortex patch embedded in a constant and uniform background shear flow. Physics Fluids A 2, 123-126.

SAto, K. (1999). Lévy Processes and Infinitely Divisible Distributions. Cambridge University Press.

Wentzell, A. D. (1990). Limit Theorems on Large Deviations for Markov Stochastic Processes. Kluwer, Dordrecht. 